\title{
Making the Case for Dynamic Wireless Infrastructure Sharing: a Techno-Economic Game
}

\author{
Alessandro Lieto* $^{* \dagger}$, Ilaria Malanchini ${ }^{\dagger}$, Vinay Suryaprakash ${ }^{\dagger}$, Antonio Capone* \\ *Dipartimento di Elettronica, Informazione e Bioingegneria Politecnico di Milano, Italy \\ Email: alessandro.lieto@mail.polimi.it, antonio.capone@polimi.it \\ ${ }^{\dagger}$ Nokia Bell Labs, Stuttgart, Germany \\ Email: \{ilaria.malanchini, vinay.suryaprakash\}@ nokia-bell-labs.com
}

\begin{abstract}
Dynamic and flexible radio resource management in a multi-tenant shared infrastructure is an effective strategy to improve the quality experienced by end users. In this framework, we propose a techno-economic model that allows network operators to compete and dynamically select the quality target to deliver to their customers, while simultaneously maximizing the profit. In order to understand the willingness of the network operators in the engagement on a sharing scenario, we develop a non-cooperative game wherein the Nash Equilibria show the disposition of operators to meet the customers' requirements. At the same time, the proposed framework show how it is challenging the definition of a new business market when scarcity of resources is assumed.
\end{abstract}

\section{INTRODUCTION}

The emergence of new technologies and their applications have caused a huge growth in mobile data traffic [1]. Mobile Network Operators (MNOs) are faced with tackling this growth, resulting in an increase in their operating expenditure (OpEx) and capital expenditure (CapEx). Data revenues, however, are not keeping pace with the traffic increase [2] and MNOs, therefore, need to redefine their business modus operandi: inevitably, cost reduction remains the main driver for profitability. Infrastructure sharing, according to the Organization for Economic Co-operation and Development (OECD), promises to provide an opportunity to cope with the more stringent requirements of future networks ( $5 \mathrm{G}$ and beyond) while reducing the total expenditure [3].

Many studies analyze the validity and the advantages of several sharing options, focusing either on the economical benefits [4], [5], [6], or on the strategic modeling of infrastructure [7], [8]. One of the main concerns while considering sharing options is coping with the tradeoff between the cost savings and the level of operational autonomy of MNOs [9].

Active sharing, which entails sharing radio access network resources including the spectrum, is the most comprehensive of the sharing methods and allows a significant reduction in costs when compared to passive sharing - as reported by the OECD report [3, Pg. 65]. However, active sharing implies some kind of control on how resources are shared. A popular approach to controlling shared resources is the use of Service Level Agreements (SLAs). Today's SLAs, however, are rather long term and do not help the MNOs cope with short term fluctuations in the traffic their networks carry. Thereby, resulting in scenarios where the MNOs either posses a surfeit of resources or a lack thereof. Therefore, active sharing has to be based on a more dynamic and flexible methodology, wherein MNOs can trade the amount of resources needed to meet their customers' demands in real-time. A first step in this direction was taken in [10], where the authors propose a techno-economic model that allows dynamic (short term) pricing and allocation of network resources. Their model is based on a comprehensive sharing scenario in which multiple Mobile Virtual Network Operators (MVNOs) lease or rent the infrastructure from an Infrastructure Provider (InP). The dynamism their model permits is due to its ability to allow deviations (from an initial long term SLA) depending on the MVNOs individual budgets as well as their traffic load. However, though [10] incorporates fluctuations in traffic and the MVNOs' budgets, it neither explicitly considers the quality of services provided to the users while modeling fluctuations in traffic nor analyzes the profitability of the MVNOs. These aspects are precisely what this paper addresses: we provide a method to translate the users' willingness to pay and the quality targets of the MVNOs into time-varying resource requirements, which are in turn used by the InP while allocating network resources to the MVNOs. It is important to note that the use of long term SLAs is no longer necessary in the model we propose. This allows the MVNOs to focus on meeting the quality assurances they make to their users rather than on the amount of resources they require. This work explores the aforementioned trade-offs with the use of game theory. Despite many works try to justify the use of a game theoretic approach for infrastructure sharing, most of them only focus on the beneficial effects of the sharing. Authors in [11] and [12] investigate the cost reduction if MVNOs cooperate in taking common decisions on network deployment. [13] analyzes also the technical advantages in terms of throughput and how to guarantee fairness solutions in the game. However, none of these works highlights the necessity to define a new business system, wherein MVNOs can even take autonomous decisions, addressing the need of differentiation policies among MVNOs [14]. This is another contribute that this paper tries to provide.

The paper is organized as follows: in Section II we describe the considered scenario and the main features involved. In Section III we present the approach and the algorithm for determining a solution of the game. Then, in Section IV the achieved results are shown, and, finally, our remarks will 
conclude the paper in Section V.

\section{SYSTEM MODEL}

We consider a multi-agent scenario, where a single infrastructure provider interacts with competitive MVNOs that have to deliver data traffic to end users. We denote the set of the MVNOs by $M$, and let $K$ be the set of the active users in the network; then, let $K_{m} \subset K$ be the set of the users of the MVNO $m$. We consider the downlink in a single base station scenario wherein the decisions taken by the base station's scheduler are not directly affected by those in the neighboring base stations.

Let $U_{t h}$ be the intended utility (in terms of spectral efficiency $[\mathrm{bps} / \mathrm{Hz}]$ ) of a generic user for a specific service requested and assume that all users in the area will require the same type of data service. Given this assumption, we can estimate the Market Share (MS) of an MVNO as the percentage of users served in the considered area:

$$
\mathrm{MS}_{m}=\frac{\left|K_{m}\right|}{\sum_{m \in M}\left|K_{m}\right|} .
$$

We assume that MVNOs set their respective quality targets, $U_{t h, m}$, as a product of the intended utility $U_{t h}$ and a quality of service parameter $Q_{m}$, i.e. $U_{t h, m}=U_{t h} \cdot Q_{m}$ : this quality parameter will set the fraction of utility that the MVNO $m$ has to provide to its users w.r.t. the one they require. The utility achieved by a user $k$ at time $n$ is defined as a function of the maximum achievable rate, $r_{k}[n]$, and the number of assigned resources, $x_{k}[n]$, i.e. $U_{k}\left(x_{k}[n], r_{k}[n]\right)$. Assuming a partitioning of time in to elementary slots of duration $1 \mathrm{~ms}$, the scheduler decides to allocate a portion of the total wireless resource, $x_{k}[n] \geq 0$, at time slot $n$ to the user $k$. This assignment will depend on the service requested by the user and on the quality target that MVNOs set for the service. The average utility achieved for all the users of a given MVNO $m$ during a time window $N$ is given by

$$
U_{a c h, m}=\frac{1}{\left|K_{m}\right||N|} \sum_{n \in N} \sum_{k \in K_{m}} U_{k}\left(x_{k}[n], r_{k}[n]\right) \text {. }
$$

Then, we model the quality experienced by a user by combining the achieved utility ${ }^{1} U_{k}$ and the tariff imposed by an MVNO. As in [15], we define the acceptance probability as the probability that a user $k$ will accept a price $p$, given the achieved utility, $U_{k}$ as:

$$
A\left(p, U_{k}\right)=1-\exp \left(-C p^{-\epsilon} U_{k}^{\mu}\right),
$$

where $C$ is a normalization factor, and $\epsilon$ and $\mu$ are microeconomic parameters. With this, the MVNOs can estimate the expected return on investment as well as the profit.

Finally, we assume the existence of a single InP who is not subject to conventional market pressures. This, in turn, allows setting a fixed price per unit of resource by ignoring market driven fluctuations arising from competition between different

\footnotetext{
${ }^{1}$ Note that the explicit functional dependence described above has been dropped in the interest of notational brevity.
}

infrastructure providers. We denote by $C_{\mathrm{op}}$, the operating expenditure (OpEx), and $C_{\text {cap }}$, the capital expenditure (CapEx).

\section{FORMULATION AND ANALYSIS}

As described in the previous section, the decisions that have to be taken in the considered system are mainly two. On one hand, the InP has to decide how to allocate the resources among the MVNOs in order to maximize fairness. On the other hand, the MVNOs have to decide how to select the quality parameter $Q_{m}$ so that their own profit is maximized. While the first one is an optimization model, the second one must include the competitive behavior of the MVNOs. Therefore, in this section, we first introduce the optimal resource allocation model and then the competitive quality selection game.

\section{A. Optimal resource allocation model}

Based on the notations defined in Section II, the generic optimization problem at a base station's scheduler can be described by Equations (4a)-(4d):

$$
\begin{gathered}
\max \alpha \\
\text { s.t. } \quad \sum_{k \in K} x_{k}[n] \leq 1, \quad \forall n \in N, \\
U_{a c h, m} \leq U_{t h, m} \quad \forall m \in M, \\
\alpha \leq \frac{U_{a c h, m}}{U_{t h, m}}, \quad \forall m \in M .
\end{gathered}
$$

Unlike [16], we assume no pre-agreement among the parties on how to share resources (e.g., in terms of service level agreement) and, therefore, introduce a fairness parameter $\alpha$.

The constraint (4b) ensures that the total number of resources assigned is always less than or equal to the total number of resources available in the network. The constraint (4c) guarantees that the InP does not assign more resources to MVNOs than their requested quality targets, because MVNOs do not want to pay for something they did not ask for. Finally, equation (4d) is the proportional fairness condition: each operator will get the same proportion of the utility target (equal to $\alpha$ ), independent of the number of users and the quality factor selected. Since the goal of the InP is to maximize the parameter $\alpha$ in (4a), it guarantees fairness among MVNOs. At the same time, the scheduler of a base station is still free to select the best users in order to efficiently allocate resources. Therefore, the proposed formulation indirectly maximizes the minimum average utility of each MVNO.

This allocation scheme can lead to competition among the MVNOs for the selection of the quality parameter. Observing equation (4d), we see that MVNOs with higher utility targets and/or larger number of users will need more resources to achieve their targets. Given a shared scenario, this will also affect the quality target of the other MVNOs. Then, MVNOs need to optimize the decisions while trading off the service quality experienced by their users with the total cost of the infrastructure required. 


\section{B. Competitive quality selection game}

We model competition among MVNOs as a non-cooperative game, where the space of players is defined by $M$. As a strategy, each player can select one among the possible values of the quality parameter $Q_{m} \in\{0,0.1, \ldots, 1\}$. We assume that the space of all the possible permutations of strategies of the players is the cartesian product of the single strategies of the MVNOs, and denote it by

$$
S=\left\{\left(q_{1}, q_{2}, \ldots, q_{n}\right): q_{1}, q_{2}, \ldots, q_{n} \in Q_{m}\right\},
$$

where the $n$-tuple of strategies is defined for $M=$ $\{1,2, \ldots, n\}$. For each strategy $s \in S$, the infrastructure provider generates a different allocation denoted by $x_{k, s}[n]$, and a given user will get a strategy dependent utility denoted by $U_{k, s}\left(x_{k, s}[n], r_{k}[n]\right)$, depending on the decision taken by the base station scheduler. The objective of the MVNOs is to maximize a profit function, defined as the difference between the payments made by its users, i.e. reward function $R_{m, s}$, and the total expenditures, i.e. $B_{m, s}$, as

$$
f_{m, s}(p)=R_{m, s}(p)-B_{m, s} .
$$

1) Cost function: The InP fixes a price for a unit of resource. Hence, we can define the cost for resources usage

$$
C_{m, s}=\frac{1}{|N|} \cdot\left(C_{o p}+C_{c a p}\right) \sum_{n \in N} \sum_{k \in K_{m}} x_{k, s}[n],
$$

which represents the total amount spent by the $m$-th MVNO for the resources used in a cell given the strategy $s$.

If the amount of resources available are insufficient to satisfy the utility of each MVNO (i.e., the achieved utility of the MVNOs will be a fraction of the desired threshold $\alpha<1$ ), the InP can introduce another component of the cost to incentivize the MVNOs to help expand the capacity of the network. We call this pressure cost [10], and it corresponds to the total investment necessary for satisfying the targets of all MVNOs. $\alpha$ being a measure of the utility achieved with the available resources, we can assume that the amount of extra resources needed can be evaluated as $\frac{1-\alpha}{\alpha}$. Hence, the pressure cost for a strategy $s$ can be written as

$$
C_{\mathrm{pre}, s}=\frac{1-\alpha_{s}}{\alpha_{s}} \cdot\left(C_{o p}+C_{c a p}\right) \cdot \lambda,
$$

where the same price for a unit of resource (in terms of OpEx and CapEx) is assumed, but is scaled by a factor $\lambda>1$ representing the return on investment for the InP. From the InP point of view, this is the total amount of money the MVNOs have to collect for investing in additional capacity. It is reasonable to assume that this expansion is carried out in the long-run and the individual cost can, therefore, be split according to the market share of the MVNO.

Hence, we can assume that each operator will pay an expenditure $B_{m}$ equal to :

$$
B_{m, s}=C_{m, s}+C_{\mathrm{pre}, s} \cdot \mathrm{MS}_{m}, \quad \forall m \in M .
$$

It is important to recall that $\alpha$ decreases with an increase in the number of users as well as an increase in the selected
$Q_{m}$ value. In the game, we can also figure out if MVNOs - at the equilibrium - are willing to invest in expanding the infrastructure or not.

2) Reward function: In our framework, we model user behavior by evaluating an acceptance probability function that matches the utility received and the price submitted into a satisfaction function for the users. Actually, for introducing the investment incentive for MVNOs, we need to define a more complex reward function, that allows the operators to estimate the advantages or disadvantages of additional investments. For this purpose, the reward function

$$
R_{m, s}(p)=\sum_{k \in K_{m}} p \cdot A\left(p, U_{k, s}\right)+F_{k, s}(p),
$$

contains two terms representing two payment criteria:

i) $p A\left(p, U_{k, s}\right)$ describing a major part of the price according to the achieved utility, in order to measure the actual satisfaction of the user, where the "optimal" price $p$ is evaluated as the one maximizing the total profit;

ii) $F_{k, s}(p)$ denoting an extra component, that varies according to the quality target promised by the MVNO.

The term in i) is the reference acceptance probability presented in Section II, so we investigate the term in ii) given by

$$
F_{k, s}(p)=Q_{m} \cdot \delta \cdot p \cdot A\left(p+Q_{m} \delta p, U_{t h} Q_{m}\right) .
$$

We assume that MVNOs can charge the users an extra fee that depends on the quality target they promise to deliver to the users. We consider the extra fee to be equal to the product of a percentage $\delta$ of the fixed price $p$ and the quality factor $Q_{m}$ selected. Then, we estimate the willingness of users to pay this cost $\left(p+Q_{m} \delta p\right)$ according to the promised utility $\left(U_{t h} Q_{m}\right)$.

Equation (10) plays a slightly different role based on the two following cases:

1) If the resources available in the network are sufficient to achieve the MVNO's threshold ( $\alpha=1)$, this term is a way to incentivize MVNOs to push for higher quality for users (short-term investment);

2) If the InP cannot fulfill the requests of the MVNOs (i.e, $\alpha<1$ ), the MVNOs need to estimate a potential return on investment for addressing a capacity expansion cost defined earlier (long-term investment).

We characterize the solution of the game by introducing the Nash Equilibrium (NE) in pure strategies [17].

\section{Applied algorithm}

The formulation described so far points out different goals for the agents involved in this scenario. On one hand, the InP optimizes the resources to allocate by maximizing the proportional fairness parameter $\alpha$ based on the MVNOs' target utility. On the other hand, the MVNOs try to maximize their profit by analyzing the user satisfaction for any given strategy.

Given this contraposition, we decouple the two objective functions (i.e., those solved by InP and the MVNOs) and solve a two-stage problem as shown in Fig. 1. This procedure 


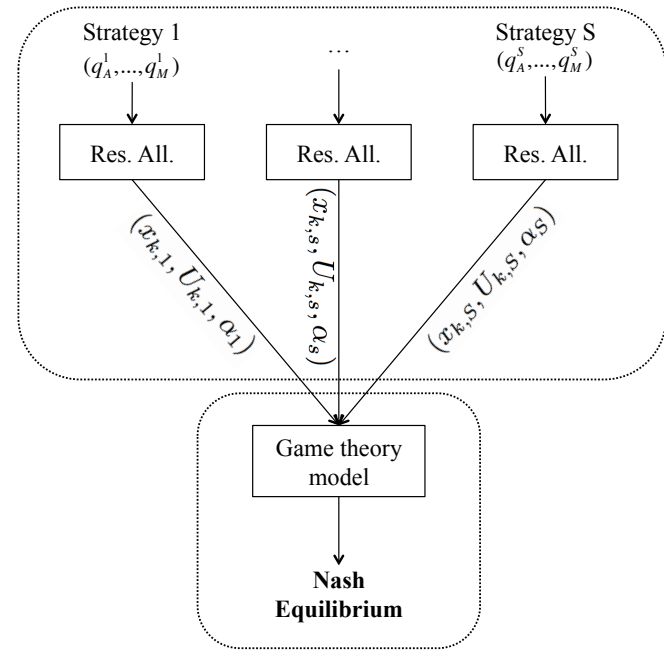

Fig. 1: Two stage optimization problem.

allows us to avoid non-linearities in the formulation, i.e. in (4d), and in the evaluation of the pressure cost. We solve $|S|$ independent optimization problems, where $|S|$ denotes the cardinality of the set of the strategies $S$. We consider all possible permutations of strategies $s$ as instances of the resource allocation problem and for each of these, we evaluate the cost function described in (8), the reward function in (9), and, lastly, the strategy dependent profit function defined in (5). Finally, we solve an optimization problem to find a NE of the game that, while optimizing the selection of the price $p$, maximizes the profit for each MVNO $m$. The existence of an equilibrium has not been proven in this framework; however, we always get a feasible solution, resulting, at least, in one equilibrium point.

\section{Simulation Results}

In this section, we analyze the MVNOs' strategies at the equilibrium and compare the results at the equilibrium with other strategies (not NE) maximizing the overall profit function.

\section{A. Simulation setup}

We consider the single base station scenario described in Section II and model the competition among three MVNOs, i.e. $|M|=3$. We also assume all the MVNOs have the same number of users and they have the same market share, i.e. $\mathrm{MS}_{m}=0.33$. The users are uniformly distributed throughout the coverage area of the base station and they are simultaneously active in each time slot of the window of observation of length $N=200$ time slots, where each time slot is of duration $1 \mathrm{~ms}$.

We model the wireless channel by a frequency-flat block fading channel with i.i.d. Rayleigh coefficients - resulting in exponentially distributed random channel gains $\left|h_{k}[n]\right|^{2}$. Then, we assume that the power received by a generic user is calculated through the Okumura-Hata model, indicating by $P$ the base station transmitted power (in Watts [W]), and by $\beta$ the
TABLE I: The applied parameters and their values.

\begin{tabular}{|c|c|c|}
\hline Parameter & Definition & Value \\
\hline \hline$C_{\mathrm{cap}}$ & CapEx Cost & 20 \\
\hline$C_{\mathrm{op}}$ & OpEx Cost & 20 \\
\hline$U_{\mathrm{th}}$ & User expected utility & $0.5 \mathrm{Mbps} / \mathrm{Hz}$ \\
\hline$\delta$ & Portion of extra price & 0.1 \\
\hline$\lambda$ & InP return on investment & 1.5 \\
\hline$p$ & Tariff/month & {$[0.3,0.6, \ldots, 30]$} \\
\hline$|M|$ & Number of MVNOs & 3 \\
\hline $\mathrm{MS}_{m}$ & Market Share & 0.33 \\
\hline$N$ & Duration of simulation & $200 \mathrm{~ms}$ \\
\hline
\end{tabular}

path-loss exponent. Hence, the average Signal-to-Interferenceplus-Noise-Ratio (SINR) of user's $k, \mathrm{SINR}_{k}$, can be computed as:

$$
\operatorname{SINR}_{k}=\frac{P \cdot d_{k}^{-\beta}}{\sigma^{2}+I_{0}},
$$

where $d_{k}$ is the user's distance from the base station (in meters $[\mathrm{m}]), \sigma^{2}$ is the thermal noise, $I_{0}$ is the average interference power of the neighboring base stations.

Due to the fast fading components, the instantaneous SINR at time slot $n$ is equal to

$$
\gamma_{k}[n]=\left|h_{k}[n]\right|^{2} \cdot \operatorname{SINR}_{k},
$$

which leads to the evaluation of the spectral efficiency of a user $k$ (in bit/s/Hz) at any time instance $n$ as

$$
r_{k}[n]=\log _{2}\left(1+\gamma_{k}[n]\right) .
$$

The utility targets are defined in terms of spectral efficiency; hence, we design the utility achieved, $U_{k}$, as a linear function of the assigned resources and the maximum achievable rate:

$$
U_{k}\left(x_{k}[n], r_{k}[n]\right)=x_{k}[n] \cdot r_{k}[n] .
$$

It is important to note that all the costs as well as the pricing tariffs are to be considered purely illustrative (see Table I). They are used for the sole purposes of understanding the key characteristic behavior of the model.

\section{B. Results}

We consider two different user densities: first, we analyze the strategies played at the equilibrium when the number of users per MVNO $K_{m}=4$, and then, when $K_{m}=15$. We observed that in both cases the NE strategy selected by the MVNOs is the one that maximizes the quality of service delivered to their users, denoted by the triple $(1,1,1)$. The symmetry of the NE strategy comes also from the symmetry in MS.

In Fig. 2, we compare the costs incurred and the profits of individual MVNOs both when the InP can and cannot fulfill the MVNOs' requirements. In Fig. 2(a), the resources available in the network are sufficient to satisfy the target utility of the MVNOs as indicated by the zero-values of the pressure cost. This condition justifies the selection based on maximum quality, i.e. based on users being completely satisfied, and the MVNOs can, therefore, maximize their profit by selecting the optimal price indicated in Fig. 3(a). 


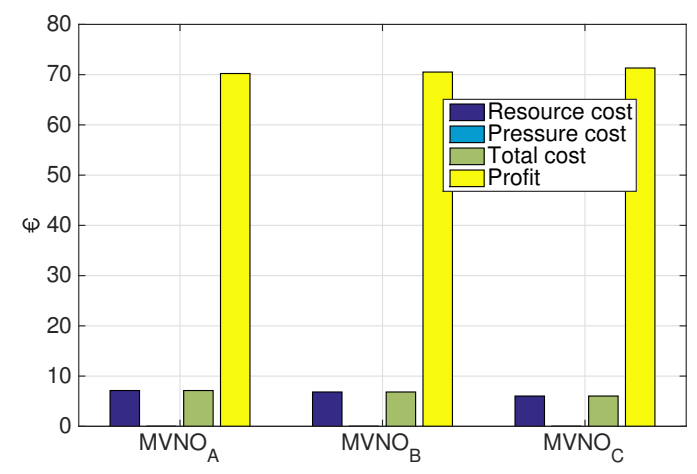

(a) 4 users per MVNO

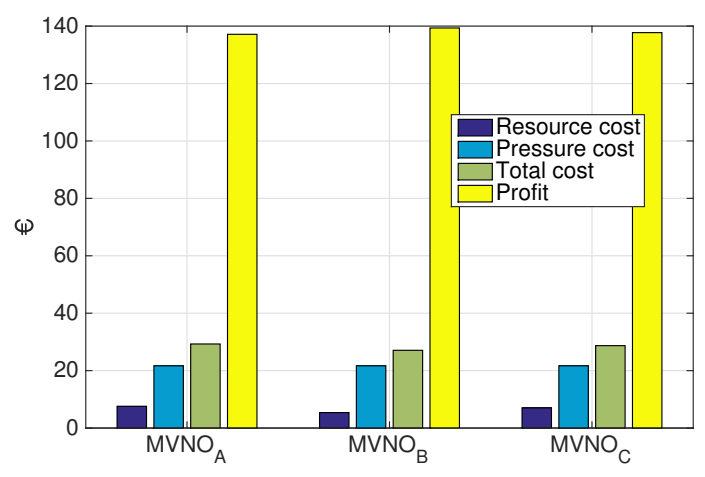

(b) 15 users per MVNO

Fig. 2: Costs and profit of each MVNO at the equilibrium strategy for different densities.

However, when the total number of users in the cell is increased, the resources are insufficient to meet the target utility. So unlike the previous case, we see an increase in the pressure cost (Fig. 2(b)), which is shared equally among the MVNOs owing to the symmetry of the problem. A consequence of resource scarcity is the decrease in the average utility of the users, $U_{k}$; this compels MVNOs to adjust the price, as shown in Fig. 3(b), in order to maximize profit ${ }^{2}$.

As shown in Fig. 4, the MVNOs could obtain higher profits by selecting a different set of strategies, which do not result in a NE such as the triple $(0.4,0.4,0.4)$. But, since the pressure cost is proportional to the market share and not the chosen quality (cf. (8)), each MVNO tries to get an advantage of this scenario, trying to maximize profit by selecting higher quality levels for its users. Hence, as result of the competitive structure, the MVNOs reach an equilibrium point by deciding to jointly invest in the network expansion.

Fig. 5 shows how the NE strategy and future investment (i.e., pressure cost) vary while increasing the number of users per MVNO. We observe that there exists a point (approximately at 40 users per MVNO) where the model is unable to sufficiently incentivize additional investments and this leads

\footnotetext{
${ }^{2}$ The increase in the profit is due to the assumption made on the selected parameter. The absolute values of the profit are not investigated in this work.
}

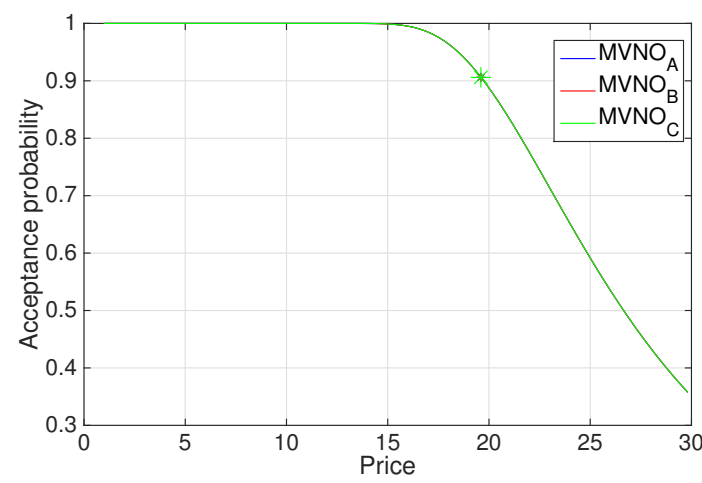

(a) 4 users per MVNO

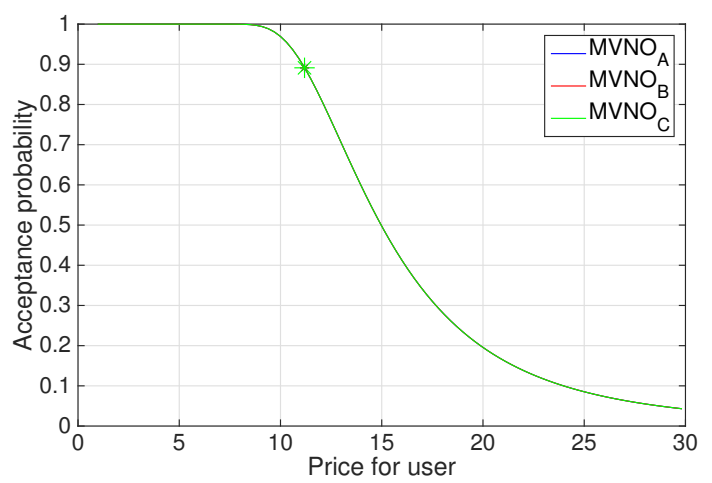

(b) 15 users per MVNO

Fig. 3: Selected optimal price: the slope of the curves changes according to the utility achieved. The optimal price is indicated by the cross intercepting the curve.

the MVNOs to select a NE strategy different from $(1,1,1)$. In other words, the resources available in the network are so scarce that additional investments are unwarranted. This implies that there exists a minimum amount of resources that the InP needs to provide such that the MVNOs can be incentivized to invest in the infrastructure. Further insights are given by the Price of Stability (PoS), defined as the ratio between the profit earned at the $\mathrm{NE}$ and the strategy maximizing the individual profit [18]. We observe that the PoS decreases while the number of users increases, which means that the profit for the MVNOs at the equilibrium strategy decreases w.r.t. the optimal one. However, when the MVNOs decide to reduce their investment, the PoS starts increasing again.

\section{CONCLuSion}

In this paper, we propose an approach to analyze the engagement of multiple MVNOs in a sharing infrastructure scenario. This work enables competition introducing a differentiation criterion among the MVNOs that are free to select the level of quality of service that they want to deliver to their users. MVNOs can estimate the economical advantages of the decision, balancing the costs addressed to the usage of the infrastructure and the profit earned from the users. 


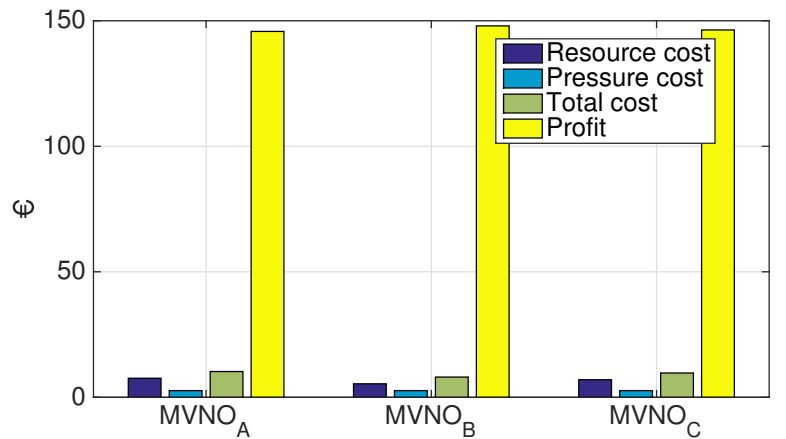

Fig. 4: Costs and profit at non-equilibrium strategy for $K_{m}=$ 15. MVNOs could maximize the profit selecting the triple $(0.4,0.4,0.4)$.
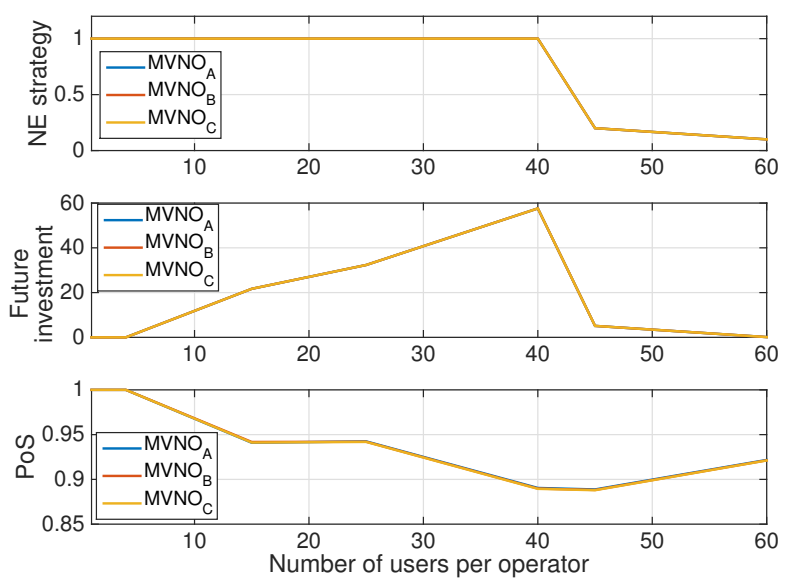

Fig. 5: Nash equilibria increasing user densities.

This framework also evaluates the willingness of the MVNOs to invest in the shared infrastructure. Assuming scarcity of resources, the competition scenario leads the MVNOs to push in the investment, although it does not represent the strategy that maximizes profit. This model also finds a limit point, in which the amount of extra resources needed is too costly to justify an investment. This may lead to the evaluation of a more complex business ecosystem wherein the competition among different InPs could find out a way to incentivize also InPs in the investment in order to meet user requirements.

\section{ACKNOWLEDGEMENT}

The work has been partially supported by the Bell Labs Internship program and by the European Union Horizon 2020 Marie Sklodowska-Curie ITN/EID project ACT5G (grant agreement No. 643002).

\section{REFERENCES}

[1] CISCO, "Cisco Visual Networking Index: Forecast and Methodology, 2015-2020 white paper," 2015. [Online]. Available: http://www.cisco.com/c/en/us/solutions/collateral/service-provider/ visual-networking-index-vni/complete-white-paper-c11-481360.pdf
[2] McKinsey and C. Inc., "Monetizing mobile: making data pay," 2014. [Online]. Available: http://cs404620.vk.me/u11927290/docs/ c94e7ac85fc9/1_McKinsey_Monetizing_mobile_2014-06.pdf

[3] OECD, "Wireless market structures and network sharing," 2014 [Online]. Available: http://dx.doi.org/10.1787/5jxt46dz19r2-en

[4] Y.-K. Song, H. Zo, and S. Lee, "Analyzing the economic effect of mobile network sharing in korea," ETRI Journal, vol. 34, no. 3, pp. 308-318, 2012.

[5] F. Berkers, G. Hendrix, I. Chatzicharistou, T. De Haas, and D. Hamera, "To share or not to share?" in Intelligence in Next Generation Networks (ICIN), 2010 14th International Conference on, Oct 2010.

[6] D.-E. Meddour, T. Rasheed, and Y. Gourhant, "On the role of infrastructure sharing for mobile network operators in emerging markets," Computer Networks, vol. 55, no. 7, pp. 1576-1591, 2011.

[7] N. Alliance, "Suggestions on potential solutions to c-ran," White Paper, January, 2013.

[8] V. Suryaprakash, P. Rost, and G. Fettweis, "Are heterogeneous cloudbased radio access networks cost effective?" IEEE Journal on Selected Areas in Communications, vol. 33, no. 10, pp. 2239-2251, Oct 2015.

[9] T. Frisanco, P. Tafertshofer, P. Lurin, and R. Ang, "Infrastructure sharing for mobile network operators; from a deployment and operations view," in 2008 International Conference on Information Networking, Jan 2008, pp. $1-5$.

[10] O. U. Akgül, I. Malanchini, V. Suryaprakash, and A. Capone, "Dynamic resource allocation and pricing for shared radio access infrastructure," in submitted to IEEE International Conference on Communications (ICC), 2017.

[11] A. Antonopoulos, E. Kartsakli, A. Bousia, L. Alonso, and C. Verikoukis, "Energy-efficient infrastructure sharing in multi-operator mobile networks," IEEE Communications Magazine, vol. 53, no. 5, pp. 242249, May 2015.

[12] L. Cano, A. Capone, G. Carello, M. Cesana, and M. Passacantando, "Cooperative infrastructure and spectrum sharing in heterogeneous mobile networks," IEEE Journal on Selected Areas in Communications, vol. 34, no. 10 , pp. 2617-2629, Oct 2016.

[13] I. Malanchini, M. Cesana, and N. Gatti, "Network selection and resource allocation games for wireless access networks," IEEE Transactions on Mobile Computing, vol. 12, no. 12, pp. 2427-2440, Dec 2013.

[14] I. Malanchini and M. Gruber, "How operators can differentiate through policies when sharing small cells," in 2015 IEEE 81st Vehicular Technology Conference (VTC Spring), May 2015.

[15] L. Badia, M. Lindstrom, J. Zander, and M. Zorzi, "Demand and pricing effects on the radio resource allocation of multimedia communication systems," in IEEE Global Telecommunications Conference, 2003. GLOBECOM '03, vol. 7, Dec 2003, pp. 4116-4121.

[16] I. Malanchini, S. Valentin, and O. Aydin, "Wireless resource sharing for multiple operators: Generalization, fairness, and the value of prediction," Computer Networks, vol. 100, pp. 110 - 123, 2016.

[17] J. Nash, "Non-cooperative games," Annals of Mathematics, vol. 54, no. 2, pp. 286-295, 1951.

[18] E. Anshelevich, A. Dasgupta, J. Kleinberg, E. Tardos, T. Wexler, and T. Roughgarden, "The price of stability for network design with fair cost allocation," in 45th Annual IEEE Symposium on Foundations of Computer Science, Oct 2004, pp. 295-304. 